\title{
NO DIALGEBRA HAS GELFAND-KIRILLOV DIMENSION STRICTLY BETWEEN 1 AND $2^{\ddagger}$
}

\author{
ZERUI ZHANG*, YUQUN CHEN ${ }^{\sharp}$, AND BING YU ${ }^{\dagger}$
}

\begin{abstract}
The Gelfand-Kirillov dimension measures the asymptotic growth rate of algebras. For every associative dialgebra $\mathcal{D}$, the quotient $\mathcal{A}_{\mathcal{D}}:=\mathcal{D} / \operatorname{Id}(S)$, where $\operatorname{Id}(S)$ is the ideal of $\mathcal{D}$ generated by the set $S:=\{x \vdash y-x \dashv y \mid x, y \in \mathcal{D}\}$, is called the associative algebra associated to $\mathcal{D}$. Here we show that the Gelfand-Kirillov dimension of $\mathcal{D}$ is bounded above by twice the Gelfand-Kirillov dimension of $\mathcal{A}_{\mathcal{D}}$. Moreover, we prove that no associative dialgebra has Gelfand-Kirillov dimension strictly between 1 and 2 .
\end{abstract}

\section{INTRODUCTION}

The Gelfand-Kirillov dimension has become one of the important tools in the study of algebras. There are many well known results on Gelfand-Kirillov dimensions of associative algebras and modules [3]. For instance, the Gelfand-Kirillov dimension of a finitely generated commutative algebra $\mathcal{A}$ over a field is the classical Krull dimension of $\mathcal{A}[3$, Theorem 4.5]. In particular, the Gelfand-Kirillov dimension of the free commutative algebra generated by $n$ elements is exactly $n$, where $n$ is a positive integer. Here we shall investigate the Gelfand-Kirillov dimensions of associative dialgebras.

Recall that an associative dialgebra (dialgebra for short) $(\mathcal{D}, \vdash, \dashv)$ over a field $k$ is a $k$ vector space equipped with two bilinear operations $\vdash: \mathcal{D} \otimes \mathcal{D} \rightarrow \mathcal{D}$ and $\dashv: \mathcal{D} \otimes \mathcal{D} \rightarrow \mathcal{D}$ such that $(\mathcal{D}, \vdash)$ and $(\mathcal{D}, \dashv)$ are associative algebras and the following identities hold:

$$
\left\{\begin{array}{l}
x \dashv(y \vdash z)=x \dashv(y \dashv z), \\
(x \dashv y) \vdash z=(x \vdash y) \vdash z, \\
x \vdash(y \dashv z)=(x \vdash y) \dashv z,
\end{array}\right.
$$

for all $x, y$ and $z$ in $\mathcal{D}$. For instance, let $(\mathcal{A}, \partial)$ be a differential associative algebra satisfying $\partial^{2}=0$, and define $x \vdash y=\partial(x) y$ and $x \dashv y=x \partial(y)$. Then $(\mathcal{A}, \vdash, \dashv)$ becomes a dialgebra [4].

Let $\mathcal{D}$ be a dialgebra and let $\mathcal{A}_{\mathcal{D}}=\mathcal{D} / \operatorname{ld}(S)$, where $\operatorname{ld}(S)$ is the ideal of $\mathcal{D}$ generated by the set $S:=\{x \vdash y-x \dashv y \mid x, y \in \mathcal{D}\}$. Then $\mathcal{A}_{\mathcal{D}}$ is an associative algebra, which is called the

1991 Mathematics Subject Classification. 16S15, 16P90, 17A30.

Key words and phrases. associative algebra; dialgebra; Gelfand-Kirillov dimension.

$¥$ Supported by the NNSF of China (11571121), the NSF of Guangdong Province (2017A030313002) and the Science and Technology Program of Guangzhou (201707010137).

* Supported by the Innovation Project of Graduate School of South China Normal University.

$\sharp$ Corresponding author. 
associative algebra associated to $\mathcal{D}$. We find that the Gelfand-Kirillov dimension $\operatorname{GKdim}(\mathcal{D})$ of $\mathcal{D}$ and the Gelfand-Kirillov dimension $\operatorname{GKdim}\left(\mathcal{A}_{\mathcal{D}}\right)$ of $\mathcal{A}_{\mathcal{D}}$ have the following relation:

Theorem A. Let $\mathcal{D}$ be a dialgebra and let $\mathcal{A}_{\mathcal{D}}$ be the associative algebra associated to $\mathcal{D}$. Then we have

$$
\operatorname{GKdim}\left(\mathcal{A}_{\mathcal{D}}\right) \leq \operatorname{GKdim}(\mathcal{D}) \leq 2 \mathrm{GKdim}\left(\mathcal{A}_{\mathcal{D}}\right)
$$

In particular, the inequality $\operatorname{GKdim}(\mathcal{D})<\infty$ holds if and only if $\operatorname{GKdim}\left(\mathcal{A}_{\mathcal{D}}\right)<\infty$ holds.

For every associative algebra $(\mathcal{A}, \cdot)$, we define $x \vdash y=x \dashv y=x \cdot y$ for all $x, y$ in $\mathcal{A}$. Then it is clear that $(\mathcal{A}, \vdash,-)$ becomes a dialgebra. It is well-known that for every real number $r \geq 2$, there exists an associative algebra $\mathcal{A}$ satisfying $\operatorname{GKdim}(\mathcal{A})=r$, so for every real number $r \geq 2$, there exists a dialgebra $\mathcal{D}$ satisfying $\operatorname{GKdim}(\mathcal{D})=r$. Bergman [1] proved that there is no associative algebra having Gelfand-Kirillov dimension in the open interval $(1,2)$. C. Martinez and E. Zelmanov [5] proved an analogous theorem for Jordan algebras. However, for Lie algebras and for Jordan superalgebras, the situations are quite different: the Gelfand-Kirillov dimension of a finitely generated Lie algebra [6] or of a Jordan superalgebra [7] can be an arbitrary number in $\{0\} \cup[1, \infty]$. This leaves the following problem open: Does there exist a dialgebra of Gelfand-Kirillov dimension in the open interval $(1,2)$ ? Based on Bergman's result, we prove the following result:

Theorem B. No dialgebra has Gelfand-Kirillov dimension strictly between 1 and 2.

Although Theorem B is based on the corresponding result of Bergman, the extension is not obvious because really different techniques are needed here, among which constructing connected linear bases for $\mathcal{D}$ and $\mathcal{A}_{\mathcal{D}}$ simultaneously plays an important role. In general, assuming $\operatorname{GKdim}\left(\mathcal{A}_{\mathcal{D}}\right)=1$ does not imply $\operatorname{GKdim}(\mathcal{D})=1$. We shall provide a sufficient condition in Lemma 3.11 ensuring $\operatorname{GKdim}(\mathcal{D})=\operatorname{GKdim}\left(\mathcal{A}_{\mathcal{D}}\right)$. Moreover, we shall show that, for $\operatorname{GKdim}\left(\mathcal{A}_{\mathcal{D}}\right)=1$, the sufficient condition is satisfied.

The paper is organized as follows. In Section 2, we recall some basic properties of a dialgebra and the definition of the Gelfand-Kirillov dimension. We then provide a formula, involving the generators of a dialgebra, for calculating the Gelfand-Kirillov dimension (Lemma 2.4). In Section 3, we introduce how to construct a shortest-middlelexicographic (linear) basis of a dialgebra (Definition 3.3). Such (linear) bases turn out to be very useful in connecting the Gelfand-Kirillov dimension of a dialgebra and that of its associated associative algebra, and they play important roles in the proofs of Theorem A and Theorem B. Finally, to prove Theorem B, we also need to investigate the middle entries (Definition 2.1) of monomials in the constructed shortest-middle-lexicographic (linear) bases.

\section{The Gelfand-Kirillov dimension of a Dialgebra}

Our aim in this section is to recall the notion of the Gelfand-Kirillov dimension of a dialgebra. We shall first recall some basic notations and properties of a dialgebra. Then we shall introduce several formulas for the Gelfand-Kirillov dimension of a dialgebra. 
Recall that for every dialgebra $\mathcal{D}$, for all $x_{1}, \ldots, x_{t}$ in $\mathcal{D}$, every parenthesizing of

$$
x_{1} \vdash \cdots \vdash x_{m} \dashv \cdots \dashv x_{t}
$$

gives the same element in $\mathcal{D}$ [4], which we denote by $\left[x_{1} \ldots x_{t}\right]_{m}$ or $x_{1} \vdash \cdots \vdash x_{m} \dashv \cdots \dashv x_{t}$. The following definition of middle submonomials of a monomial resembles that of subwords of a word.

Definition 2.1. [4] For all $x_{1}, \ldots, x_{t}$ in $\mathcal{D}$, the element $x_{m}$ in the monomoial $\left[x_{1} \ldots x_{t}\right]_{m}$ is called the middle entry of $\left[x_{1} \ldots x_{t}\right]_{m}$, and every monomial $\left[x_{p} \ldots x_{q}\right]_{m-p+1}$ satisfying $1 \leq p \leq$ $m \leq q \leq t$ is called a middle submonomial of $\left[x_{1} \ldots x_{t}\right]_{m}$.

The following formulas for calculating the product of two monomials in a dialgebra will be very useful in the sequel. Roughly speaking, the middle entry of the product of two monomials is the middle entry that the operation $\vdash$ or $\dashv$ points to. More precisely, we have the following lemma:

Lemma 2.2. [4] For all integers $m, n, p, q$ such that $1 \leq p \leq n<m$ and $1 \leq q \leq m-n$, for all $x_{1}, \ldots, x_{m}$ in a dialgebra $\mathcal{D}$, we have the following formulas:

(i) $\left[x_{1} \ldots x_{n}\right]_{p} \vdash\left[x_{n+1} \ldots x_{m}\right]_{q}=\left[x_{1} \ldots x_{m}\right]_{n+q}$.

(ii) $\left[x_{1} \ldots x_{n}\right]_{p} \dashv\left[x_{n+1} \ldots x_{m}\right]_{q}=\left[x_{1} \ldots x_{m}\right]_{p}$.

Before introducing a general definition of the Gelfand-Kirillov dimension of a dialgebra $\mathcal{D}$ over a field $k$, we have to introduce some more notations. Let $V, V_{1}$ and $V_{2}$ be vector subspaces of $\mathcal{D}$. We first define

$$
V_{1} \vdash V_{2}=\operatorname{span}_{k}\left\{x \vdash y \mid x \in V_{1}, y \in V_{2}\right\} \text { and } V_{1} \dashv V_{2}=\operatorname{span}_{k}\left\{x \dashv y \mid x \in V_{1}, y \in V_{2}\right\} .
$$

Then we define $V^{1}=V$ and $V^{n}=\sum_{1 \leq i \leq n-1}\left(V^{i} \vdash V^{n-i}+V^{i} \dashv V^{n-i}\right)$ for every integer number $n \geq 2$. Finally, we define

$$
V^{\leq n}:=V^{1}+V^{2}+\cdots+V^{n}
$$

By Lemma 2.2, we easily obtain

$$
V^{n}=\operatorname{span}_{k}\left\{\left[x_{1} \ldots x_{n}\right]_{p} \mid 1 \leq p \leq n, \text { where } n, p \in \mathbb{N} \text { and } x_{1}, \ldots, x_{n} \in V\right\}
$$

and

$$
V^{\leq n}=\operatorname{span}_{k}\left\{\left[x_{1} \ldots x_{m}\right]_{p} \mid 1 \leq p \leq m \leq n, \text { where } p, m, n \in \mathbb{N} \text { and } x_{1}, \ldots, x_{m} \in V\right\} .
$$

Now we are ready to introduce the Gelfand-Kirillov dimension of a dialgebra.

Definition 2.3. Let $\mathcal{D}$ be a dialgebra over a field $k$, the Gelfand-Kirillov dimension of a dialgebra $\mathcal{D}$ is defined to be

$$
\operatorname{GKdim}(\mathcal{D})=\sup _{V} \varlimsup_{n \rightarrow \infty} \log _{n} \operatorname{dim}\left(V^{\leq n}\right),
$$

where the supremum is taken over all finite dimensional subspaces $V$ of $\mathcal{D}$. 
For instance, let $\mathcal{D}$ be the free dialgebra generated by a letter $a$. Then it is easy to see that $\left\{\left[a_{1} \ldots a_{n}\right]_{p} \mid a_{1}=\cdots=a_{n}=a, 1 \leq p \leq n\right.$, and $\left.p, n \in \mathbb{N}\right\}$ is a linear basis of $\mathcal{D}$. So by direct calculation, we obtain $\operatorname{GKdim}(\mathcal{D})=2$.

By Definition 2.3, if $\mathcal{D}^{\prime}$ is a subalgebra of $\mathcal{D}$ or a homomorphic image of $\mathcal{D}$, then we have $\operatorname{GKdim}\left(\mathcal{D}^{\prime}\right) \leq \operatorname{GKdim}(\mathcal{D})$. And since we often consider generating sets of a dialgebra, we shall also use the following formula for calculating the Gelfand-Kirillov dimension in the sequel.

Lemma 2.4. Let $\mathcal{D}$ be a dialgebra generated by a set $X$. Then we have

$$
\operatorname{GKdim}(\mathcal{D})=\sup _{X^{\prime} \subseteq X} \varlimsup_{n \rightarrow \infty} \log _{n} \operatorname{dim}\left(\left(k X^{\prime}\right)^{\leq n}\right),
$$

where the supremum is taken over all finite nonempty subsets $X^{\prime}$ of $X$, and $k X^{\prime}$ is the vector subspace of $\mathcal{D}$ spanned by $X^{\prime}$.

Proof. Let $V$ be a finite dimensional subspace of $\mathcal{D}$. Then for some finite subset $X^{\prime}$ of $X$ and for some integer number $m$, we have $V \subseteq\left(k X^{\prime}\right)^{\leq m}$ and $V^{\leq n} \subseteq\left(\left(k X^{\prime}\right)^{\leq m}\right)^{\leq n} \subseteq\left(k X^{\prime}\right)^{\leq m n}$ for every integer number $n \geq 1$. Consequently, we deduce

$$
\begin{aligned}
\varlimsup_{n \rightarrow \infty} \log _{n} \operatorname{dim}\left(V^{\leq n}\right) & \leq \varlimsup_{n \rightarrow \infty} \log _{n} \operatorname{dim}\left(\left(k X^{\prime}\right)^{\leq m n}\right) \stackrel{t=m n}{=} \frac{\lim _{t \rightarrow \infty} \frac{\log _{t} \operatorname{dim}\left(\left(k X^{\prime}\right)^{\leq t}\right)}{\log _{t}(t / m)}}{} \\
& =\varlimsup_{t \rightarrow \infty} \log _{t} \operatorname{dim}\left(\left(k X^{\prime}\right)^{\leq t}\right) \leq \sup _{X^{\prime} \subseteq X^{\prime}} \varlimsup_{t \rightarrow \infty} \log _{t} \operatorname{dim}\left(\left(k X^{\prime}\right)^{\leq t}\right)
\end{aligned}
$$

and

$$
\operatorname{GKdim}(\mathcal{D})=\sup _{V} \varlimsup_{n \rightarrow \infty} \log _{n} \operatorname{dim}\left(V^{\leq n}\right) \leq \sup _{X^{\prime} \subseteq X} \varlimsup_{t \rightarrow \infty} \log _{t} \operatorname{dim}\left(\left(k X^{\prime}\right)^{\leq t}\right) .
$$

On the other hand, for every finite subset $X^{\prime}$ of $X$, the subspace $k X^{\prime}$ is a finite dimensional subspace of $\mathcal{D}$. By Definition 2.3, the lemma follows.

As a consequence, when one applies Equation (2.1) to calculate the Gelfand-Kirillov dimension of a dialgebra $\mathcal{D}$, it is independent of the choices of the sets of generators of $\mathcal{D}$. In particular, when $\mathcal{D}$ is finitely generated, Equation (2.1) becomes easier:

Corollary 2.5. Let $\mathcal{D}$ be a dialgebra generated by a finite set $X$. Then we have

$$
\operatorname{GKdim}(\mathcal{D})=\varlimsup_{n \rightarrow \infty} \log _{n} \operatorname{dim}\left((k X)^{\leq n}\right) .
$$

In particular, for every dialgebra $\mathcal{D}$, we have $\operatorname{GKdim}(\mathcal{D})=\sup _{\mathcal{D}^{\prime}} \operatorname{GKdim}\left(\mathcal{D}^{\prime}\right)$, where the supremum is taken over all finitely generated subalgebras $\mathcal{D}^{\prime}$ of $\mathcal{D}$.

\section{No dialgebra has Gelfand-Kirillov dimension in the open interval $(1,2)$}

Our aim in this section is to prove our main results on Gelfand-Kirillov dimensions of dialgebras. We shall prove the inequality $\operatorname{GKdim}\left(\mathcal{A}_{\mathcal{D}}\right) \leq \operatorname{GKdim}(\mathcal{D}) \leq 2 \operatorname{GKdim}\left(\mathcal{A}_{\mathcal{D}}\right)$ and construct dialgebras such that $\operatorname{GKdim}\left(\mathcal{A}_{\mathcal{D}}\right)=\operatorname{GKdim}(\mathcal{D})$ or $\operatorname{GKdim}(\mathcal{D})=2 \operatorname{GKdim}\left(\mathcal{A}_{\mathcal{D}}\right)$. Finally, we shall conclude the paper with Theorem B.

We first recall the linear basis of a free dialgebra generated by an arbitrary well-ordered set $X$ constructed by Loday [4]. Let $(X,<)$ be a fixed well-ordered set. Then we use $X^{+}$for 
the free semigroup generated by $X$. For every $u=a_{1} \ldots a_{n}$ in $X^{+}$, where $a_{1}, \ldots, a_{n}$ lie in $X$, we define the length $\ell(u)$ of $u$ to be $n$. Finally, let $\mathcal{D}(X)$ be the free dialgebra generated by $X$. Then the following set

$$
\left[X^{+}\right]_{\omega}:=\left\{\left[a_{1} \ldots a_{n}\right]_{m} \mid a_{1}, \ldots, a_{n} \in X, m, n \in \mathbb{N}, 1 \leq m \leq n\right\}
$$

forms a linear basis of $\mathcal{D}(X)$ [4]. For a nonempty sequence $u=a_{1} \ldots a_{n}$ over $X$, we shall also use the notation $[u]_{m}$ for $\left[a_{1} \ldots a_{n}\right]_{m}$, and we call $\left[a_{1} \ldots a_{n}\right]_{m}$ a disequence over $X$. Obviously, two disequences $\left[a_{1} \ldots a_{n}\right]_{m}$ and $\left[b_{1} \ldots b_{p}\right]_{q}$ are the same only if $n=p, m=q$ and $a_{i}=b_{i}$ for every $i \leq n$. Finally, for a subset $Y$ of $\left[X^{+}\right]_{\omega}$, we define $|Y|$ to be cardinality of the set $Y$.

Now we recall a well-ordering on $\left[X^{+}\right]_{\omega}$ introduced in [2]. Here we use a slightly different name for the ordering to make it easy to remember how we compare two monomials.

Definition 3.1. [2] We recall that the length-middle-lexicographic ordering $<$ on $\left[X^{+}\right]_{\omega}$ is defined as follows: For all $[u]_{p}=\left[a_{1} \ldots a_{n}\right]_{p}$ and $[v]_{q}=\left[b_{1} \ldots b_{m}\right]_{q}$ in $\left[X^{+}\right]_{\omega}$ such that $a_{1}, \ldots, a_{n}$ and $b_{1}, \ldots, b_{m}$ lie in $X$, we define

$$
[u]_{p}<[v]_{q} \text { if }\left(\ell(u), p, a_{1}, \ldots, a_{n}\right)<\left(\ell(v), q, b_{1}, \ldots, b_{m}\right) \text { lexicographically. }
$$

For instance, for all $a_{1}, \ldots, a_{4}$ in $X$ such that $a_{1}<\cdots<a_{4}$, we have $\left[a_{4} a_{3} a_{2}\right]_{1}<\left[a_{1} a_{3} a_{2}\right]_{2}$ and $\left[a_{1} a_{2} a_{3} a_{4}\right]_{1}>\left[a_{1} a_{2} a_{4}\right]_{2}$. Note that the above ordering is not compatible with the products in general. For instance, we have $\left[a_{1} a_{2}\right]_{2}>\left[a_{2} a_{1}\right]_{1}$ and $\left[a_{1} a_{2}\right]_{2} \vdash a_{3}<\left[a_{2} a_{1}\right]_{1} \vdash a_{3}$.

However, the length-middle-lexicographic ordering still has some good properties as follows:

Lemma 3.2. Let $\left[u_{1}\right]_{m_{1}},\left[u_{2}\right]_{m_{2}}$ and $\left[u_{3}\right]_{m_{3}}$ be monomials in $\left[X^{+}\right]_{\omega}$ with $\left[u_{1}\right]_{m_{1}}<\left[u_{2}\right]_{m_{2}}$. Then we have $\left[u_{3}\right]_{m_{3}} \vdash\left[u_{1}\right]_{m_{1}}<\left[u_{3}\right]_{m_{3}} \vdash\left[u_{2}\right]_{m_{2}}$ and $\left[u_{1}\right]_{m_{1}} \dashv\left[u_{3}\right]_{m_{3}}<\left[u_{2}\right]_{m_{2}} \dashv\left[u_{3}\right]_{m_{3}}$; if, in addition, $m_{2}=1$, then we also have

$$
\left[u_{3}\right]_{m_{3}} \dashv\left[u_{1}\right]_{m_{1}}<\left[u_{3}\right]_{m_{3}} \dashv\left[u_{2}\right]_{m_{2}} \text { and }\left[u_{1}\right]_{m_{1}} \vdash\left[u_{3}\right]_{m_{3}}<\left[u_{2}\right]_{m_{2}} \vdash\left[u_{3}\right]_{m_{3}} .
$$

Proof. Note that $\left[u_{1}\right]_{m_{1}}<\left[u_{2}\right]_{1}$ implies $\left(\ell\left(u_{1}\right), m_{1}\right) \leq\left(\ell\left(u_{2}\right), 1\right)$. Moreover, if $\ell\left(u_{1}\right)=\ell\left(u_{2}\right)$, then we obtain $m_{1}=1$. The remain of the proof is straightforward.

Let $\mathcal{D}$ be a dialgebra generated by a well-ordered set $X$. Then $\left[X^{+}\right]_{\omega}$ is a linear generating set of $\mathcal{D}$. Moreover, there is no harm to assume that $\mathcal{A}_{\mathcal{D}}$ is also generated by $X$ and $\left[X^{+}\right]_{\omega}$ is also a linear generating set of $\mathcal{A}_{\mathcal{D}}$. To avoid possible confusions, whenever we write down a linear combination $f$ of elements in $\left[X^{+}\right]_{\omega}$, we shall declare $f$ in $\mathcal{D}$ or $\mathcal{A}_{\mathcal{D}}$. In this way, we are able to use the same notation for an element in $\mathcal{D}$ or in $\mathcal{A}_{\mathcal{D}}$.

We are now ready to introduce a general way of constructing a subset $B_{\mathcal{D}}$ of $\left[X^{+}\right]_{\omega}$ for a dialgebra $\mathcal{D}$ with respect to a generating set $X$, and we shall see in Lemma 3.4 that $B_{\mathcal{D}}$ turns out to be a linear basis of $\mathcal{D}$. By convention, we shall assume that the empty set is a linear basis of the trivial vector space $\{0\}$.

Definition 3.3. Let $\mathcal{D}$ be a dialgebra generated by a well-ordered set $X$. We call

$B_{\mathcal{D}}:=\left\{[u]_{p} \in\left[X^{+}\right]_{\omega} \mid\right.$ for all integer number $n$, for all $\left[u_{1}\right]_{p_{1}}, \ldots,\left[u_{n}\right]_{p_{n}}$ in $\left[X^{+}\right]_{\omega}$ such that $\left[u_{i}\right]_{p_{i}}<[u]_{p}$ for every $i \leq n$, the element $[u]_{p}$ can not be written as a linear combination of the elements $\left[u_{1}\right]_{p_{1}}, \ldots,\left[u_{n}\right]_{p_{n}}$ in $\mathcal{D}$ and $[u]_{p}$ is not 0 in $\left.\mathcal{D}\right\}$ 
the shortest-middle-lexicographic basis of $\mathcal{D}$ with respect to $X$.

By Definition 3.3, we know that $B_{\mathcal{D}}$ depends on the generating set $X$. However, to make the notation and formulas simple, we shall still use the notation $B_{\mathcal{D}}$ but not $B_{\mathcal{D}, X}$. (No confusions arise in the sequel.)

Lemma 3.4. Let $\mathcal{D}$ be a dialgebra generated by a well-ordered set $X$. Then the shortestmiddle-lexicographic basis $B_{\mathcal{D}}$ of $\mathcal{D}$ with respect to $X$ is a linear basis of $\mathcal{D}$. In particular, let $\mathcal{A}=\mathcal{A}_{\mathcal{D}}$ be the associative algebra associated to $\mathcal{D}$, and let $B_{\mathcal{A}}$ be the shortest-middlelexicographic basis of $\mathcal{A}$ with respect to $X$. Then $B_{\mathcal{A}}$ is a linear basis of $\mathcal{A}$.

Proof. If $\mathcal{D}=\{0\}$, then $B_{\mathcal{D}}$ is an empty set. Now we assume $\mathcal{D} \neq\{0\}$. To show that $B_{\mathcal{D}}$ is a linear generating set of $\mathcal{D}$, it suffices to show that every $[u]_{p}$ in $\left[X^{+}\right]_{\omega}$ lies in the subspace $\operatorname{span}_{k}\left(B_{\mathcal{D}}\right)$ of $\mathcal{D}$ spanned by $B_{\mathcal{D}}$. We use induction on $[u]_{p}$ with respect to the length-middle-lexicographic ordering in Definition 3.1. The minimal element of $X$ that is not 0 in $\mathcal{D}$, say $a$, lies in $B_{\mathcal{D}}$ by the definition of $B_{\mathcal{D}}$. Suppose $[u]_{p}>a$. If $[u]_{p}$ lies in $B_{\mathcal{D}}$ or $[u]_{p}=0$ in $\mathcal{D}$, then there is nothing to prove. Otherwise, say $[u]_{p}=\sum_{1<i<n} \alpha_{i}\left[u_{i}\right]_{p_{i}}$ for some elements $\alpha_{1}, \ldots, \alpha_{n}$ in $k$ and for some monomials $\left[u_{1}\right]_{p_{1}}, \ldots,\left[u_{n}\right]_{p_{n}}$ in $\left[\bar{X}^{+}\right]_{\omega}$ such that $\left[u_{i}\right]_{p_{i}}<[u]_{p}$ for every $i \leq n$, then by induction hypothesis, we have $[u]_{p} \in \operatorname{span}_{k}\left(B_{\mathcal{D}}\right)$. Finally, by the construction of $B_{\mathcal{D}}$, it is clear that the set $B_{\mathcal{D}}$ is linear independent in $\mathcal{D}$.

Now we show that, every $x \neq 0$ in $\mathcal{D}$ such that $x=0$ in $\mathcal{A}_{\mathcal{D}}$ is a left zero divisor with respect $\vdash$ and a right zero divisor with respect to $\dashv$ in $\mathcal{D}$. To simplify the formulas, we define $\varepsilon$ to be an empty disequence, and for every $y$ in $\mathcal{D}$, we define $\varepsilon \vdash y=y \dashv \varepsilon=y$.

Lemma 3.5. Let $\mathcal{D}$ be a dialgebra and let $\mathcal{A}_{\mathcal{D}}$ be the associative algebra associated to $\mathcal{D}$. Then for every $x$ in $\mathcal{D}$ such that $x=0$ in $\mathcal{A}_{\mathcal{D}}$, for every $y$ in $\mathcal{D}$, we have $x \vdash y=y \dashv x=0$ in $\mathcal{D}$.

Proof. If $x=0$ in $\mathcal{A}_{\mathcal{D}}$, then we have $x=\sum \alpha_{j}\left(z_{j} \vdash\left(x_{j} \vdash y_{j}-x_{j} \dashv y_{j}\right)\right) \dashv z_{j}^{\prime}$ in $\mathcal{D}$ by Lemma 2.2, where each $x_{j}$ and each $y_{j}$ lie in $\mathcal{D}$, each $z_{j}$ and each $z_{j}^{\prime}$ may be elements of $\mathcal{D}$ or be the empty disequence $\varepsilon$. So for every $y$ in $\mathcal{D}$, by Lemma 2.2 again, we obtain

$$
\begin{aligned}
& \left(\left(z_{j} \vdash\left(x_{j} \vdash y_{j}-x_{j} \dashv y_{j}\right)\right) \dashv z_{j}^{\prime}\right) \vdash y \\
= & \left(\left(z_{j} \vdash\left(x_{j} \vdash y_{j}\right)\right) \dashv z_{j}^{\prime}\right) \vdash y-\left(\left(z_{j} \vdash\left(x_{j} \dashv y_{j}\right)\right) \dashv z_{j}^{\prime}\right) \vdash y \\
= & \left(\left(z_{j} \vdash\left(x_{j} \vdash y_{j}\right)\right) \dashv z_{j}^{\prime}\right) \vdash y-\left(\left(z_{j} \vdash\left(x_{j} \vdash y_{j}\right)\right) \dashv z_{j}^{\prime}\right) \vdash y=0 .
\end{aligned}
$$

Similar to the above reasoning, we obtain $y \dashv x=0$.

Before going further, we shall observe some more properties on the shortest-middlelexicographic basis of a dialgebra. In Lemma 3.6, we shall use the shortest-middlelexicographic basis of an associative subalgebra $\mathcal{A}^{\prime}$ of $\mathcal{A}_{\mathcal{D}}$ generated by $X^{\prime}$, where $X^{\prime}$ is a nonempty subset of $X$. By changing every occurrence of $\mathcal{D}$ into $\mathcal{A}^{\prime}$ and every occurrence of $X$ into $X^{\prime}$ in (3.1), we shall obtain the corresponding set $B_{\mathcal{A}^{\prime}}$.

Lemma 3.6. Let $\mathcal{D}$ be a dialgebra generated by a well-ordered set $X$, and let $\mathcal{A}=\mathcal{A}_{\mathcal{D}}$ be the associative algebra associated to $\mathcal{D}$. Let $\mathcal{D}^{\prime}$ be a subalgebra of $\mathcal{D}$ generated by 
a subset $X^{\prime}$ of $X$, and let $\mathcal{A}^{\prime}$ be a subalgebra of $\mathcal{A}$ generated by $X^{\prime}$. Then for every monomial $\left[a_{1} \ldots a_{t}\right]_{p}$ in the shortest-middle-lexicographic basis $B_{\mathcal{A}^{\prime}}$ of $\mathcal{A}^{\prime}$ with respect to $X^{\prime}$, where the letters $a_{1}, \ldots, a_{t}$ lie in $X^{\prime}$, we have $p=1$. Moreover, if $\left[a_{1} \ldots a_{t}\right]_{p}$ lies in the shortest-middle-lexicographic basis $B_{\mathcal{D}^{\prime}}$ of $\mathcal{D}^{\prime}$ with respect to $X^{\prime}$ and $p>1$, then $\left[a_{1} \ldots a_{p-1}\right]_{1}$ lies in $B_{\mathcal{A}^{\prime}}$; if $\left[a_{1} \ldots a_{t}\right]_{p}$ lies in $B_{\mathcal{D}^{\prime}}$ and $p<t$, then $\left[a_{p+1} \ldots a_{t}\right]_{1}$ lies in $B_{\mathcal{A}^{\prime}}$.

Proof. By the definition of $\mathcal{A}$, for every integer number $p$ such that $1 \leq p \leq t$, we have $\left[a_{1} \ldots a_{t}\right]_{p}=\left[a_{1} \ldots a_{t}\right]_{1}$ in $\mathcal{A}$ and thus in $\mathcal{A}^{\prime}$. So $\left[a_{1} \ldots a_{t}\right]_{p} \in B_{\mathcal{A}^{\prime}}$ forces $p=1$. For the second claim, we may assume that $p>1$ without loss of generality. Suppose that $\left[a_{1} \ldots a_{p-1}\right]_{1} \notin B_{\mathcal{A}^{\prime}}$.

If $\left[a_{1} \ldots a_{p-1}\right]_{1}=0$ in $\mathcal{A}^{\prime}$ and thus in $\mathcal{A}$, then by Lemma 3.5, we have

$$
\left[a_{1} a_{2} \cdots a_{t}\right]_{p}=\left[a_{1} \ldots a_{p-1}\right]_{1} \vdash\left[a_{p} \ldots a_{t}\right]_{1}=0 \text { in } \mathcal{D} \text { and hence in } \mathcal{D}^{\prime},
$$

which contradicts with the fact that $\left[a_{1} \ldots a_{t}\right]_{p}$ lies in $B_{\mathcal{D}^{\prime}}$.

If $\left[a_{1} \ldots a_{p-1}\right]_{1} \neq 0$ in $\mathcal{A}^{\prime}$, then we have $\left[a_{1} \ldots a_{p-1}\right]_{1}=\sum_{1 \leq i \leq n} \alpha_{i}\left[u_{i}\right]_{p_{i}}$ in $\mathcal{A}^{\prime}$ and thus in $\mathcal{A}$ for some monomials $\left[u_{1}\right]_{p_{1}}, \ldots,\left[u_{n}\right]_{p_{n}}$ in $\left[X^{\prime+}\right]_{\omega}$ satisfying $\left[u_{i}\right]_{p_{i}}<\left[a_{1} \ldots a_{p-1}\right]_{1}$ for every integer $i \leq n$ and for some elements $\alpha_{1}, \ldots, \alpha_{n}$ in the field $k$. In particular, by Lemma 3.5 again, we obtain

$\left[a_{1} \ldots a_{t}\right]_{p}-\sum_{1 \leq i \leq n} \alpha_{i}\left[u_{i}\right]_{p_{i}} \vdash\left[a_{p} a_{p+1} \ldots a_{t}\right]_{1}=\left(\left[a_{1} \ldots a_{p-1}\right]_{1}-\sum_{1 \leq i \leq n} \alpha_{i}\left[u_{i}\right]_{p_{i}}\right) \vdash\left[a_{p} a_{p+1} \ldots a_{t}\right]_{1}=0$

in $\mathcal{D}$, and thus in $\mathcal{D}^{\prime}$. Finally, by Lemma 3.2 , we obtain $\left[u_{i}\right]_{p_{i}} \vdash\left[a_{p} a_{p+1} \ldots a_{t}\right]_{1}<\left[a_{1} \ldots a_{t}\right]_{p}$, which contradicts with the construction of $B_{\mathcal{D}^{\prime}}$.

Note that the length-middle-lexicographic ordering on $\left[X^{+}\right]_{\omega}$ plays an important role in Lemma 3.6. Using another way of constructing linear bases for $\mathcal{D}$ and $\mathcal{A}_{\mathcal{D}}$ simultaneously may not have the claimed properties in the lemma.

Remark 3.7. With the notations of Lemma 3.6, if $X \neq X^{\prime}$, then $\mathcal{A}^{\prime}$ is not necessary the associative algebra associated to $\mathcal{D}^{\prime}$ in general. For instance, let $X=\{a, b\}$, and let $\mathcal{D}(a, b)$ be the free dialgebra generated by $X$. Then we define $\mathcal{D}$ to be the quotient of $\mathcal{D}(a, b)$ by the ideal generated by the set $\{b=a \vdash a-a \dashv a\}$. It is clear that $\mathcal{D}$ is isomorphic to the free dialgebra generated by $\{a\}$ and $\mathcal{A}$ is isomorphic to the free associative algebra generated by $\{a\}$. Finally, for $X^{\prime}=\{b\}$, we deduce that, $\mathcal{D}^{\prime}$ and the associative algebra associated to $\mathcal{D}^{\prime}$ are the linear space spanned by $b$ while $\mathcal{A}^{\prime}$ is the 0 space.

Let $\mathcal{D}$ be a dialgebra generated by a finite well-ordered set $X$ and let $B_{\mathcal{D}}$ be the shortestmiddle-lexicographic basis of $\mathcal{D}$ with respect to $X$. For every positive integer $n$, we define

$$
B_{\mathcal{D}}^{n}=\left\{[u]_{p} \in B_{\mathcal{D}} \mid \ell(u)=n\right\}, B_{\mathcal{D}}^{\leq n}=\left\{[u]_{p} \in B_{\mathcal{D}} \mid \ell(u) \leq n\right\} .
$$

Then we have $\operatorname{dim}\left((k X)^{\leq n}\right)=\left|B_{\overline{\mathcal{D}}}^{\leq n}\right|$ for every $n \geq 1$, where $\left|B_{\overline{\mathcal{D}}}^{\leq n}\right|$ is the cardinality of the set $B_{\overline{\mathcal{D}}}^{\leq n}$. In particular, by Corollary 2.5, when $\mathcal{D}$ is generated by a finite well-ordered set $X$, we deduce the following formula for calculating the Gelfand-Kirillov dimension of $\mathcal{D}$ :

$$
\operatorname{GKdim}(\mathcal{D})=\varlimsup_{n \rightarrow \infty} \log _{n}\left|B_{\mathcal{D}}^{\leq n}\right| .
$$


Now we are ready to prove Theorem A of the introduction.

Theorem 3.8. Let $\mathcal{D}$ be a dialgebra and let $\mathcal{A}_{\mathcal{D}}$ be the associative algebra associated to $\mathcal{D}$. Then we have

$$
\operatorname{GKdim}\left(\mathcal{A}_{\mathcal{D}}\right) \leq \operatorname{GKdim}(\mathcal{D}) \leq 2 \mathrm{GKdim}\left(\mathcal{A}_{\mathcal{D}}\right)
$$

In particular, the inequality $\operatorname{GKdim}(\mathcal{D})<\infty$ holds if and only if $\operatorname{GKdim}\left(\mathcal{A}_{\mathcal{D}}\right)<\infty$ holds.

Proof. We use the notations of Lemma 3.6. By Definition 2.3, it is easy to see that, for every homomorphic image $\mathcal{D}_{1}$ of $\mathcal{D}$, we have $\operatorname{GKdim}\left(\mathcal{D}_{1}\right) \leq \operatorname{GKdim}(\mathcal{D})$. In particular, we obtain $\operatorname{GKdim}\left(\mathcal{A}_{\mathcal{D}}\right) \leq \operatorname{GKdim}(\mathcal{D})$.

Now we turn to the inequality $\operatorname{GKdim}(\mathcal{D}) \leq 2 \operatorname{GKdim}\left(\mathcal{A}_{\mathcal{D}}\right)$. For finitely generated algebras $\mathcal{D}^{\prime}$ and $\mathcal{A}^{\prime}$ as in Lemma 3.6, we define $\left|B_{\mathcal{A}^{\prime}}^{0}\right|=\left|B_{\mathcal{D}^{\prime}}^{0}\right|=1$ for convenience. Then by Lemma 3.6, for every integer number $t \geq 1$, we obtain $\left|B_{\mathcal{D}^{\prime}}^{t}\right| \leq \sum_{1 \leq p \leq t}\left|B_{\mathcal{A}^{\prime}}^{p-1}\right|\left|X^{\prime}\right|\left|B_{\mathcal{A}^{\prime}}^{t-p}\right|$ and

$$
\begin{aligned}
& \quad\left|B_{\mathcal{D}^{\prime}}^{\leq n}\right|=\sum_{1 \leq t \leq n}\left|B_{\mathcal{D}^{\prime}}^{t}\right| \leq \sum_{1 \leq t \leq n} \sum_{1 \leq p \leq t}\left|B_{\mathcal{A}^{\prime}}^{p-1}\right|\left|X^{\prime}\right|\left|B_{\mathcal{A}^{\prime}}^{t-p}\right|=\left|X^{\prime}\right| \sum_{\substack { 1 \leq t \leq n \\
\begin{subarray}{c}{i \geq 0 ; j \geq 0 ; \\
i+j=t-1{ 1 \leq t \leq n \\
\begin{subarray} { c } { i \geq 0 ; j \geq 0 ; \\
i + j = t - 1 } }\end{subarray}}\left|B_{\mathcal{A}^{\prime}}^{i}\right|\left|B_{\mathcal{A}^{\prime}}^{j}\right| \\
& =\left|X^{\prime}\right| \sum_{0 \leq i \leq n-1}\left(\left|B_{\mathcal{A}^{\prime}}^{i}\right| \sum_{0 \leq j \leq n-1-i}\left|B_{\mathcal{A}^{\prime}}^{j}\right|\right) \leq\left|X^{\prime}\right| \sum_{0 \leq i \leq n-1}\left(\left|B_{\mathcal{A}^{\prime}}^{i}\right|\left(\left|B_{\mathcal{A}^{\prime}}^{\leq n-1}\right|+\left|B_{\mathcal{A}^{\prime}}^{0}\right|\right)\right) \\
& \leq\left|X^{\prime}\right|\left(\left|B_{\mathcal{A}^{\prime}}^{\leq n-1}\right|+\left|B_{\mathcal{A}^{\prime}}^{0}\right|\right) \sum_{0 \leq i \leq n-1}\left|B_{\mathcal{A}^{\prime}}^{i}\right| \leq\left|X^{\prime}\right|\left(\left|B_{\mathcal{A}^{\prime}}^{\leq n-1}\right|+\left|B_{\mathcal{A}^{\prime}}^{0}\right|\right)^{2} \leq\left|X^{\prime}\right|\left(\left|B_{\mathcal{A}^{\prime}}^{\leq n}\right|+1\right)^{2} .
\end{aligned}
$$

Consequently, we obtain

$\operatorname{GKdim}\left(\mathcal{D}^{\prime}\right)=\varlimsup_{n \rightarrow \infty} \log _{n}\left|B_{\mathcal{D}^{\prime}}^{\leq n}\right| \leq \varlimsup_{n \rightarrow \infty} \log _{n}\left(\left|X^{\prime}\right|\left(\left|B_{\mathcal{A}^{\prime}}^{\leq n}\right|+1\right)^{2}\right)=2 \operatorname{GKdim}\left(\mathcal{A}^{\prime}\right) \leq 2 \operatorname{GKdim}\left(\mathcal{A}_{\mathcal{D}}\right)$.

By Corollary 2.5, we deduce $\operatorname{GKdim}(\mathcal{D})=\sup _{\mathcal{D}^{\prime}} \operatorname{GKdim}\left(\mathcal{D}^{\prime}\right) \leq 2 \operatorname{GKdim}\left(\mathcal{A}_{\mathcal{D}}\right)$, where the supremum is taken over all finitely generated subalgebras $\mathcal{D}^{\prime}$ of $\mathcal{D}$.

Corollary 3.9. Let $\mathcal{D}$ be a dialgebra. Then $\operatorname{GKdim}(\mathcal{D})<1$ implies that $\operatorname{GKdim}(\mathcal{D})=0$.

Proof. Let $\mathcal{A}_{\mathcal{D}}$ be the associative algebra associated to $\mathcal{D}$. Then $\operatorname{GKdim}(\mathcal{D})<1$ implies that $\operatorname{GKdim}\left(\mathcal{A}_{\mathcal{D}}\right)=0$. So we obtain $0=\operatorname{GKdim}\left(\mathcal{A}_{\mathcal{D}}\right) \leq \operatorname{GKdim}(\mathcal{D}) \leq 2 \operatorname{GKdim}\left(\mathcal{A}_{\mathcal{D}}\right)=0$.

Now we construct examples to show that the boundaries in Theorem 3.8 are the best that one can expect. We shall describe a sufficient condition ensuring $\operatorname{GKdim}(\mathcal{D})=\operatorname{GKdim}\left(\mathcal{A}_{\mathcal{D}}\right)$ in Lemma 3.11.

Example 3.10. Let $\mathcal{D}$ be the free dialgebra generated by a letter $a$, and let $\mathcal{A}_{\mathcal{D}}$ be the associative algebra associated to $\mathcal{D}$. Then we have $\operatorname{GKdim}(\mathcal{D})=2 \operatorname{GKdim}\left(\mathcal{A}_{\mathcal{D}}\right)=2$.

Proof. Note that $B_{\mathcal{D}}=\left\{\left[a_{1} \ldots a_{n}\right]_{p} \mid a_{1}=\cdots=a_{n}=a, 1 \leq p \leq n\right.$, and $\left.p, n \in \mathbb{N}\right\}$, and $\mathcal{A}_{\mathcal{D}}$ is the free commutative algebra generated by $a$. We have $\operatorname{GKdim}(\mathcal{D})=2 \operatorname{GKdim}\left(\mathcal{A}_{\mathcal{D}}\right)=2$ immediately by Equation (3.2). 
Lemma 3.11. Let $\mathcal{D}$ be a dialgebra generated by a finite well-ordered set $X$, and let $\mathcal{A}_{\mathcal{D}}$ be the associative algebra associated to $\mathcal{D}$. If there exists a positive integer $m$ such that $\mathcal{D}$ has a linear generating set $B=\left\{\left[a_{1} \ldots a_{n}\right]_{p} \mid a_{1}, \ldots, a_{n} \in X, 1 \leq p \leq m\right.$ or $0 \leq n-p \leq m-1$, where $p, n$ lies in $\mathbb{N}$ and $p \leq n\}$. Then we obtain $\operatorname{GKdim}(\mathcal{D})=\operatorname{GKdim}\left(\mathcal{A}_{\mathcal{D}}\right)$.

Proof. For simplicity, we denote $\mathcal{A}_{\mathcal{D}}$ by $\mathcal{A}$. Since $\operatorname{GKdim}(\mathcal{A}) \leq \operatorname{GKdim}(\mathcal{D})$, it suffices to show $\operatorname{GKdim}(\mathcal{D}) \leq \operatorname{GKdim}(\mathcal{A})$. Let $B_{\mathcal{A}}$ be the shortest-middle-lexicographic basis of $\mathcal{A}$ with respect to $X$ and let $B_{1}$ be the subset of $B$ defined as follows

$$
B_{1}=\left\{\left[a_{1} \ldots a_{n}\right]_{p} \in B \mid\left[a_{1} \ldots a_{p-1}\right]_{1} \in B_{\mathcal{A}} \text { if } p>1 \text {, and }\left[a_{p+1} \ldots a_{n}\right]_{1} \in B_{\mathcal{A}} \text { if } p<n\right\} .
$$

We claim that $B_{1}$ is a linear generating set of $\mathcal{D}$. We use induction with respect to the length-middle-lexicographic ordering on $\left[X^{+}\right]_{\omega}$ to show that every element $\left[a_{1} \ldots a_{n}\right]_{p}$ of $B$ can be written as a linear combination of elements of $B_{1}$. It is clear that $X$ is a subset of $B_{1}$. Without loss of generality, we assume that $p>1$ and $\left[a_{1} \ldots a_{p-1}\right]_{1} \notin B_{\mathcal{A}}$. The following proof is similar to Lemma [3.6. If $\left[a_{1} \ldots a_{p-1}\right]_{1}=0$ in $\mathcal{A}$, then we have $\left[a_{1} \ldots a_{n}\right]_{p}=0$ in $\mathcal{D}$. If $\left[a_{1} \ldots a_{p-1}\right]_{1} \neq 0$ in $\mathcal{A}$, then we may assume that $\left[a_{1} \ldots a_{p-1}\right]_{1}=\sum_{1 \leq i \leq n} \alpha_{i}\left[u_{i}\right]_{p_{i}}$ in $\mathcal{A}$ for some monomials $\left[u_{1}\right]_{p_{1}}, \ldots,\left[u_{n}\right]_{p_{n}}$ in $\left[X^{+}\right]_{\omega}$ satisfying $\left[u_{i}\right]_{p_{i}}<\left[a_{1} \ldots a_{p-1}\right]_{1}$ for every integer $i$ such that $1 \leq i \leq n$ and for some elements $\alpha_{1}, \ldots, \alpha_{n}$ in the field $k$. By Lemma 3.2, we obtain $\left[u_{i}\right]_{p_{i}} \vdash\left[a_{p} a_{p+1} \cdots a_{n}\right]_{1}<\left[a_{1} \ldots a_{n}\right]_{p}$. Finally, we have

$$
\left[a_{1} \ldots a_{n}\right]_{p}=\left[a_{1} \ldots a_{p-1}\right]_{1} \vdash\left[a_{p} a_{p+1} . . a_{n}\right]_{1}=\sum_{1 \leq i \leq n} \alpha_{i}\left[u_{i}\right]_{p_{i}} \vdash\left[a_{p} a_{p+1} \cdots a_{n}\right]_{1} .
$$

Since $\ell\left(u_{i}\right) \leq p-1<m$, each monomial $\left[u_{i}\right]_{p_{i}} \vdash\left[a_{p} a_{p+1} \cdots a_{n}\right]_{1}$ lies in $B$. By induction hypothesis, each monomial $\left[u_{i}\right]_{p_{i}} \vdash\left[a_{p} a_{p+1} \cdots a_{n}\right]_{1}$, and thus $\left[a_{1} \ldots a_{n}\right]_{p}$, can be written as a linear combination of elements of $B_{1}$.

For every $t \geq 1$, define $B_{1}^{t}=\left\{\left[a_{1} \ldots a_{t}\right]_{p} \in B_{1} \mid a_{1}, \ldots, a_{t} \in X, p \in \mathbb{N}\right\}, B_{1}^{\leq t}=\cup_{1 \leq i \leq t} B_{1}^{i}$ and define $\left|B_{1}^{0}\right|=1$. Then for every $t \geq 1$, we have

$$
\left|B_{1}^{t}\right| \leq \sum_{\substack{1 \leq p \leq m ; \\ p \leq t}}\left|B_{\mathcal{A}}^{p-1}\right||X|\left|B_{\mathcal{A}}^{t-p}\right|+\sum_{\substack{t-m+1 \leq p \leq t ; \\ p \geq 1}}\left|B_{\mathcal{A}}^{p-1}\right||X|\left|B_{\mathcal{A}}^{t-p}\right|=2 \sum_{\substack{1 \leq p \leq m ; \\ p \leq t}}\left|B_{\mathcal{A}}^{p-1}\right||X|\left|B_{\mathcal{A}}^{t-p}\right|,
$$

and

$$
\begin{aligned}
& \quad\left|B_{1}^{\leq n}\right|=\sum_{1 \leq t \leq n}\left|B_{1}^{t}\right| \leq 2 \sum_{\substack { 1 \leq t \leq n \\
\begin{subarray}{c}{1 \leq p \leq m ; \\
p \leq t{ 1 \leq t \leq n \\
\begin{subarray} { c } { 1 \leq p \leq m ; \\
p \leq t } }\end{subarray}}\left|B_{\mathcal{A}}^{p-1}\right||X|\left|B_{\mathcal{A}}^{t-p}\right|=2|X| \sum_{1 \leq t \leq n} \sum_{\substack{0 \leq i \leq m-1 ; j \geq 0 ; \\
i+j=t-1}}\left|B_{\mathcal{A}}^{i}\right|\left|B_{\mathcal{A}}^{j}\right| \\
& =2|X| \sum_{\substack{0 \leq i \leq m-1 ; \\
i \leq n-1}}\left(\left|B_{\mathcal{A}}^{i}\right| \sum_{0 \leq j \leq n-1-i}\left|B_{\mathcal{A}}^{j}\right|\right) \leq 2|X| \sum_{0 \leq i \leq m-1}\left(\left|B_{\mathcal{A}}^{i}\right|\left(\left|B_{\mathcal{A}}^{\leq n-1}\right|+\left|B_{\mathcal{A}}^{0}\right|\right)\right) \\
& \leq 2|X|\left(\left|B_{\mathcal{A}}^{\leq n-1}\right|+\left|B_{\mathcal{A}}^{0}\right|\right) \sum_{0 \leq i \leq m-1}\left|B_{\mathcal{A}}^{i}\right| \leq 2|X|\left(\left|B_{\mathcal{A}}^{\leq n-1}\right|+\left|B_{\mathcal{A}}^{0}\right|\right)\left(\left|B_{\mathcal{A}}^{\leq m-1}\right|+\left|B_{\mathcal{A}}^{0}\right|\right) .
\end{aligned}
$$

Finally, we obtain

$\varlimsup_{n \rightarrow \infty} \log _{n}\left|B_{1}^{\leq n}\right| \leq \varlimsup_{n \rightarrow \infty} \log _{n}\left(2|X|\left(\left|B_{\mathcal{A}}^{\leq n-1}\right|+\left|B_{\mathcal{A}}^{0}\right|\right)\left(\left|B_{\mathcal{A}}^{\leq m-1}\right|+\left|B_{\mathcal{A}}^{0}\right|\right)\right)=\varlimsup_{n \rightarrow \infty} \log _{n}\left|B_{\mathcal{A}}^{\leq n-1}\right|$. 
By Corollary 2.5, we obtain $\operatorname{GKdim}(\mathcal{D}) \leq \operatorname{GKdim}(\mathcal{A})$.

There are many dialgebras satisfying the assumptions of Lemma 3.11. In particular, certain identities ensure the validity of the assumptions of Lemma 3.11.

Corollary 3.12. Let $\mathcal{D}$ be a dialgebra generated by a finite set $X$, and let $\mathcal{A}_{\mathcal{D}}$ be the associative algebra associated to $\mathcal{D}$. If $\mathcal{D}$ satisfies one of the identities: (i) $x \vdash y=y \vdash x$ for all $x, y \in \mathcal{D}$; (ii) $x \dashv y=y \dashv x$ for all $x, y \in \mathcal{D}$; (iii) $x \vdash y=y \dashv x$ for all $x, y \in \mathcal{D}$, then we have $\operatorname{GKdim}(\mathcal{D})=\operatorname{GKdim}\left(\mathcal{A}_{\mathcal{D}}\right) \leq|X|$, in particular, $\operatorname{GKdim}(\mathcal{D})$ is a nonnegative integer.

Proof. We shall show that, if one of the identities (i)-(iii) holds, then the assumptions of Lemma 3.11 are valid. Without loss of generality, assume that identity (i) holds in $\mathcal{D}$. Then for every $\left[a_{1} \ldots a_{t}\right]_{p}$ in $\mathcal{D}$ such that $p>1$, we have

$$
\left[a_{1} \ldots a_{t}\right]_{p}=\left[a_{1} \ldots a_{p-1}\right]_{p-1} \vdash\left[a_{p} \ldots a_{t}\right]_{1}=\left[a_{p} \ldots a_{t}\right]_{1} \vdash\left[a_{1} \ldots a_{p-1}\right]_{p-1}=\left[a_{p} \ldots a_{t} a_{1} \ldots a_{p-1}\right]_{t} .
$$

In particular, the set $B_{1}=\left\{\left[a_{1} \ldots a_{n}\right]_{p} \mid a_{1}, \ldots, a_{n} \in X, p=1\right.$ or $\left.p=n, n \in \mathbb{N}\right\}$ is a linear generating set of $\mathcal{D}$. By Lemma 3.11, we obtain $\operatorname{GKdim}(\mathcal{D})=\operatorname{GKdim}\left(\mathcal{A}_{\mathcal{D}}\right)$. Moreover, since $\mathcal{A}_{\mathcal{D}}$ is a commutative algebra generated by $X$, the Gelfand-Kirillov $\operatorname{dimension} \operatorname{GKdim}\left(\mathcal{A}_{\mathcal{D}}\right)$ is a nonnegative integer satisfying $\operatorname{GKdim}\left(\mathcal{A}_{\mathcal{D}}\right) \leq|X|$.

Recall that a dialgebra $(\mathcal{D}, \vdash, \dashv)$ is commutative [8] if both $\vdash$ and $\dashv$ are commutative, that is, for all $x$ and $y$ in $\mathcal{D}$, we have $x \vdash y=y \vdash x$ and $x \dashv y=y \dashv x$.

Corollary 3.13. Let $\mathcal{D}$ be a finitely generated commutative dialgebra. Then $\operatorname{GKdim}(\mathcal{D})$ is a nonnegative integer number. In particular, if $\mathcal{D}$ is a free commutative dialgebra generated by $n$ letters, then we have $\operatorname{GKdim}(\mathcal{D})=n$.

Now we shall conclude the paper with our main result (Theorem B of the introduction), which is based on Bergman's corresponding result for associative algebras.

Theorem 3.14. No dialgebra has Gelfand-Kirillov dimension strictly between 1 and 2.

Proof. We first assume that $\mathcal{D}$ is a dialgebra generated by a finite well-ordered set $X$ satisfying the inequality $\operatorname{GKdim}(\mathcal{D})<2$, and we shall show that $\operatorname{GKdim}(\mathcal{D})=\operatorname{GKdim}\left(\mathcal{A}_{\mathcal{D}}\right)$. Let $B_{\mathcal{D}}$ be the shortest-middle-lexicographic basis of $\mathcal{D}$ with respect to $X$. Then for some positive integer $m$, we have $\left|B_{\mathcal{D}}^{m}\right|<m$, for otherwise we would have $\left|B_{\mathcal{D}}^{\leq n}\right| \geq 1+2+\cdots+n$ for all $n$ and thus $\operatorname{GKdim}(\mathcal{D}) \geq 2$. Define

$$
B=\left\{\left[a_{1} \ldots a_{t}\right]_{p} \mid a_{1}, \ldots, a_{t} \in X, p, t \in \mathbb{N}, p \leq t, 1 \leq p \leq m \text { or } 0 \leq t-p \leq m-1\right\} .
$$

We shall show that $B_{\mathcal{D}}$ is a subset of $B$ and thus by Lemma 3.11 we deduce $\operatorname{GKdim}(\mathcal{D})=$ $\operatorname{GKdim}\left(\mathcal{A}_{\mathcal{D}}\right)$.

For every $\left[a_{1} \ldots a_{t}\right]_{p}$ in $B_{\mathcal{D}}$, if $t \leq 2 m$, then it is clear that $\left[a_{1} \ldots a_{t}\right]_{p}$ lies in $B$. Assume now that $t>2 m$ and $m<p<t-m+1$, then we deduce $p+m-1<t$ and $p-(m-1)>1$. Therefore, for every integer $j$ such that $0 \leq j \leq m-1$, the monomial

$$
\left[u_{j}\right]_{m-j}:=\left[a_{p+j-(m-1)} a_{p+j-(m-1)+1} \ldots a_{p+j}\right]_{m-j}
$$


is a middle submonomial of $\left[a_{1} \ldots a_{t}\right]_{p}$, and we have $\left[u_{i}\right]_{m-i}<\left[u_{j}\right]_{m-j}$ if $i>j$. Since $\left|B_{\mathcal{D}}^{m}\right|<m$, the set $\left\{\left[u_{j}\right]_{m-j} \mid 0 \leq j \leq m-1\right\} \cup B_{\overline{\mathcal{D}}}^{\leq m-1}$ is linear dependent in $\mathcal{D}$. In other words, for some integer $n$ such that $0 \leq n \leq m-1$, we have

$$
\left[u_{n}\right]_{m-n}=-\sum_{n+1 \leq j \leq m-1} \alpha_{j}\left[u_{j}\right]_{m-j}-\sum_{1 \leq i \leq l} \beta_{i}\left[w_{i}\right]_{q_{i}}
$$

for some elements $\alpha_{n+1}, \ldots, \alpha_{m-1}, \beta_{1}, \ldots, \beta_{l}$ in $k$ and for some monomials $\left[w_{1}\right]_{q_{1}}, \ldots,\left[w_{l}\right]_{q_{l}}$ in $\left[X^{+}\right]_{\omega}$ such that $\left[w_{i}\right]_{q_{i}}<\left[u_{n}\right]_{m-n}$ for every $i \leq l$. Finally, we obtain

$$
\begin{aligned}
{\left[a_{1} \ldots a_{t}\right]_{p}=- } & \sum_{n+1 \leq j \leq m-1} \alpha_{j}\left(\left[a_{1} \ldots a_{p+n-(m-1)-1}\right]_{1} \vdash\left[u_{j}\right]_{m-j}\right) \dashv\left[a_{p+n+1} \ldots a_{t}\right]_{1} \\
& -\sum_{1 \leq i \leq l} \beta_{i}\left(\left[a_{1} \ldots a_{p+n-(m-1)-1}\right]_{1} \vdash\left[w_{i}\right]_{q_{i}}\right) \dashv\left[a_{p+n+1} \ldots a_{t}\right]_{1} .
\end{aligned}
$$

Since each $[v]_{q}$ on the right hand side of Equation (3.4) satisfies $[v]_{q}<\left[a_{1} \ldots a_{t}\right]_{p}$, we obtain a contradiction with the definition of $B_{\mathcal{D}}$. So we have $B_{\mathcal{D}} \subseteq B$ and $\operatorname{GKdim}(\mathcal{D})=\operatorname{GKdim}\left(\mathcal{A}_{\mathcal{D}}\right)$. Finally, the inequality $1 \leq \operatorname{GKdim}(\mathcal{D})<2$ forces $\operatorname{GKdim}(\mathcal{D})=\operatorname{GKdim}\left(\mathcal{A}_{\mathcal{D}}\right)=1$.

Assume now that $\mathcal{D}$ is not finitely generated. It is clear that for every finitely generated subalgebra $\mathcal{D}^{\prime}$ of $\mathcal{D}$, we have $\operatorname{GKdim}\left(\mathcal{D}^{\prime}\right) \leq \operatorname{GKdim}(\mathcal{D})<2$. By the above reasoning, we deduce $\operatorname{GKdim}\left(\mathcal{D}^{\prime}\right)=0$ or 1 . Moreover, by Corollary 2.5, we obtain $\operatorname{GKdim}(\mathcal{D}) \leq 1$. Finally, the inequality $1 \leq \operatorname{GKdim}(\mathcal{D})<2$ forces $\operatorname{GKdim}(\mathcal{D})=1$.

For summary, the set of all the possible Gelfand-Kirillov dimensions of dialgebras is $\{0,1\} \cup[2, \infty)$.

What remains open in view of the previous results is the question of whether the boundaries of inequation (3.3) are the only possible values of the Gelfand-Kirillov dimension of a dialgebra, that is, can one show that either $\operatorname{GKdim}(\mathcal{D})=\operatorname{GKdim}\left(\mathcal{A}_{\mathcal{D}}\right)$ or $\operatorname{GKdim}(\mathcal{D})=$ $2 \mathrm{GK} \operatorname{dim}\left(\mathcal{A}_{\mathcal{D}}\right)$ holds? We have a feeling that this is true, but so far, we have no proof in this direction.

\section{REFERENCES}

[1] G. M. Bergman, A note on growth functions of algebras and semigroups, Research Note, University of California, Berkeley, 1978, unpublished mimeographed notes.

[2] L. A. Bokut, Y.Q. Chen and C.H. Liu, Gröbner-Shirshov bases for dialgebras, International Journal of Algebra and Computation, 20 (2010) 391-415.

[3] G. Krause and T. Lenagan, Growth of Algebras and Gelfand-Kirillov Dimension, Graduate studies in mathematics, Vol. 22. AMS, 2000.

[4] J. -L. Loday, Dialgebras, In: Dialgebras and Related Operads, Lecture Notes in Mathematics, Vol. 1763. Springer-Verlag, 2001, 7-66.

[5] C. Martinez and E. Zelmanov, Jordan algebras of Gelfand-Kirillov dimension one, Journal of Algebra, 180 (1996) 211-238.

[6] V.M. Petrogradsky, On Lie algebras with nonintegral q-dimensions. Proc. Am. Math. Soc. 125(3) (1997) 649-656 
[7] Victor Petrogradsky, I. P. Shestakov, On Jordan doubles of slow growth of Lie superalgebras, São Paulo Journal of Mathematical Sciences, https://doi.org/10.1007/s40863019-00122-x

[8] A. V. Zhuchok, Free commutative dimonoids, Algebra Discrete Math. 9(1) (2010) 109119 .

Z.Z., School of Mathematical Sciences, South China Normal University, Guangzhou 510631, P. R. CHINA

E-mail address: 295841340@qq.com

Y.C., School of Mathematical Sciences, South China Normal University, Guangzhou 510631 , P. R. China

E-mail address: yqchen@scnu.edu.cn

Y.B., School of Mathematical Sciences, South China Normal University Guangzhou 510631, P. R. ChinA

E-mail address: 449224830@qq.com 